University of New Hampshire

University of New Hampshire Scholars' Repository

Physics Scholarship

Physics

$1-1-1989$

\title{
Magnetospheric plasma pressures in the midnight meridian: Observations from 2.5 to $35 \mathrm{RE}$
}

Harlan E. Spence

Boston University, harlan.spence@unh.edu

M. G. Kivelson

R. J. Walker

D. J. McComas

Follow this and additional works at: https://scholars.unh.edu/physics_facpub

Part of the Physics Commons

\section{Recommended Citation}

Spence, H. E., M. G. Kivelson, R. J. Walker, and D. J. McComas (1989), Magnetospheric plasma pressures in the midnight meridian: Observations from 2.5 to 35 RE, J. Geophys. Res., 94(A5),5264-5272, doi:10.1029/JA094iA05p05264.

This Article is brought to you for free and open access by the Physics at University of New Hampshire Scholars' Repository. It has been accepted for inclusion in Physics Scholarship by an authorized administrator of University of New Hampshire Scholars' Repository. For more information, please contact Scholarly.Communication@unh.edu. 


\title{
Magnetospheric Plasma Pressures in the Midnight Meridian: Observations From 2.5 to $35 \mathrm{R}_{\mathrm{E}}$
}

\author{
HARLAN E. SPENCE ${ }^{1}$, MARGARET G. KIVELSON ${ }^{1}$, AND RAYMOND J. WALKER \\ Institute of Geophysics and Planetary Physics, University of California, Las Angeles
}

DAVID J. MCCOMAS

Las Alamos National Laboratory, New Mexico

\begin{abstract}
Plasma pressure data from the ISEE 2 fast plasma experiment (FPE) were statistically analyzed to determine the plasma sheet pressure versus distance in the midnight local time sector of the near-earth (12-35 $R_{E}$ ) magnetotail plasma sheet. The observed plasma pressure, assumed isotropic, was mapped along model magnetic field flux tubes (obeained from the Tsyganenko and Usmanov [1982] model) to the magnetic equator, sorted according to magnetic activity, and binned according to the mapped equatorial location. In regions $(L \gtrsim 12$ $\mathrm{R}_{E}$ ) where the bulk of the plasma pressure was contributed by particles in the energy range of the FPE (70 eV to $40 \mathrm{keV}$ for ions), the statistically determined peak plasma pressures vary with distance similarly to previously determined lobe magnetic pressures (i.e., in a time-averaged sense, pressure balance normal to the magnetotail magnetic equator in the midnight meridian is maintained between lobe magnetic and plasma sheet plasma pressures). Additional plasma pressure dala obtained in the inner magnetosphere $(2.5<\mathrm{L}<7)$ by the Explorer 45. ATS 5, and AMPTE CCE spacecraft supplement the ISEE 2 data. Estimates of plasma pressures in the "transition" region (7-12 $\mathbf{R}_{E}$ ), where the magnetic field topology changes rapidly from a dipolar to a tail-like configuration, are compared with the observed pressure profiles. The quiet time "transition" region pressure estimates, oblained previously from inversions of empirical magnetic field models, bridge observations both interior to and exterior to the "transition" region in a reasonable manner. Quiet time observations and estimates are combined to provide profiles of the equatorial plasma pressure along the midnight meridian between 2.5 and $35 R_{E}$.
\end{abstract}

\section{INTRODUCTION}

In the quiet magnetosphere, the electromagnetic forces acting on the plasma contained within the magnetic field are expected to be in near equilibrium with gradients of the plasma pressure. Thus, the configuration of the equilibrium magnetosphere is determined by the magnetic field of the Earth, the solar wind external to the cavity and by the plasma trapped within the cavity which is an internal current source. Several global magnetospheric magnetic field models have been developed to describe the magnetic fields and current systems within the magnetospheric cavity. The best semi-empirical models, based upon a vast data base of magnetometer measurements gathered over the last two decades, provide quantitative descriptions valid over large portions of the system. Because the electrical current is proportional to the curl of the magnetic field, the models also implicitly contain information on the electromagnetic forces. Therefore if magnetohydrodynamic momentum balance is assumed, magnetic field models can be used to infer the distributions of plasma consistent with the field configuration [Walker and Southwood, 1982; Spence et al., 1987].

Modeling techniques have not been applied extensively to plasma pressure measurements principally because the data have not been sufficiently comprehensive. Some studies have characterized the plasma pressure in limited energy ranges and/or spatial regions, but models require more complete data sets. In this pa-

\footnotetext{
${ }^{1}$ Also at Department of Earth and Space Sciences, University of Califomia, Los Angeles
}

Copyright 1989 by the American Geophysical Union. per, we use ISEE 2 data to establish radial profiles of averaged plasma pressures as a function of magnetic activity in the nearmidnight magnetotail. By supplementing the ISEE 2 results with previously published results we obtain pressure profiles for radial distances from $2.5 R_{E}$ to $35 R_{E}$. Determination of the average plasma properties may contribute to the eventual synthesis of field and plasma data into a comprehensive empirical description of the magnetosphere.

\section{OBSERVATIONS}

\subsection{ISEE 2 Fast Plasma Experiment}

Data from the ISEE 2 fast plasma experiment (FPE) were analyzed to determine the average distribution of plasma pressures in the magnetotail. The ISEE 2 FPE was made up of three $90^{\circ}$ spherical section electrostatic analyzers. Two "back-to-back" detectors sampled the full two-dimensional (2D) velocity distribution of both ions and electrons during each spacecraft spin period (about $3 \mathrm{~s}$ ). The third FPE detector measured the full three dimensional (3D) distribution over a somewhat longer period (eight spin periods). Because no ion mass discrimination was available, the ion distributions were analyzed assuming that all the ions were protons. The FPE system was operated in one of two energy modes: MS/M or SW/MS. The former mode was designed for measuring the magnetosphere, magnetopause and inner magnetosheath, while the latter mode was for the solar wind, bow shock, and outer magnetosheath regions. For the present study, only data from the MS/M mode were investigated. The energy ranges covered in this mode were $70 \mathrm{eV}$ to $40 \mathrm{keV}$ for the ions and $12 \mathrm{eV}$ to $20 \mathrm{keV}$ for the electrons. The reader is urged to consult Bame et al. [1978] for a more complete description.

The ISEE 2 spacecraft was launched on October 22, 1977, into 


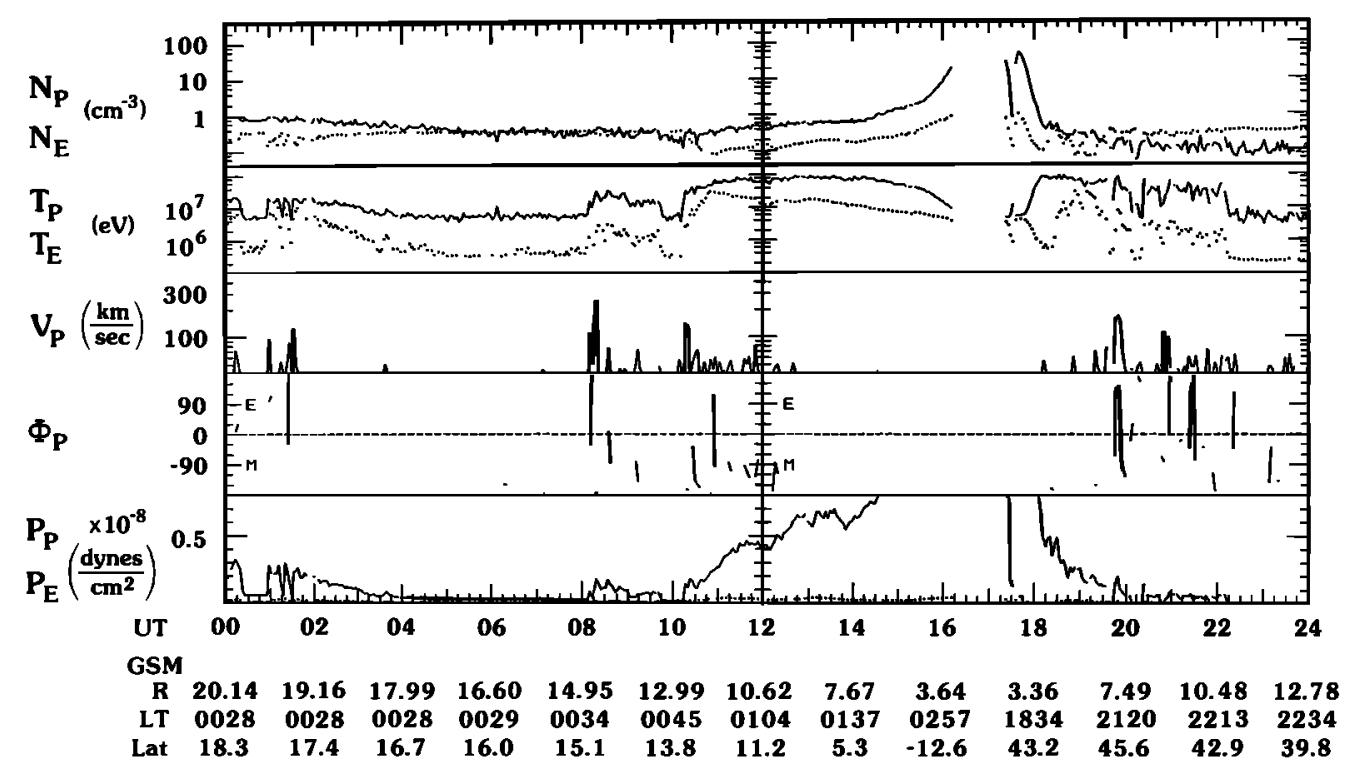

Fig. 1. An example of the 2D velocity moments derived from the FPE on ISEE 2 for April 12, 1979. Ion distributions are analyzed assuming that all ions are protons. Solid (dotted) curves are, from top to bottom, the proton (electron) number density $\left(\mathrm{cm}^{-3}\right), 2 \mathrm{D}$ temperature $(\mathrm{eV})$, flow speed $\left(\mathrm{km} \mathrm{s}^{-1}\right)$, flow azimuth (degrees), and pressure $\left(\times 10^{-8}\right.$ dynes $\left.\mathrm{cm}^{-2}\right)$. The slowly varying pressure, most clearly present as the s/c moves earthward between 1000 UT to $1400 \mathrm{UT}$, is taken to represent the steady-state plasma sheet pressure in this analysis.

an elliptical orbit with an apogee of nearly $23 R_{E}$. Slow orbital precession allowed for investigations of the magnetosphere at all local times. For the present study, we are interested in periods when the ISEE 2 spacecraft was near the midnight meridian (as defined by $\left|Y_{G S M}\right|<4 R_{E}$ ). The near-midnight meridian tail traversals occurred roughly from mid-March through early May at the beginning of the ISEE mission. Unfortunately, the FPE on ISEE 2 failed in April 1980. All relevant data from the three intervals with spacecraft apogee in the magnetotail are included in our study (1978, 1979, and 1980).

The goal of this study is to combine all the near-tail FPE plasma pressure data to determine how the average pressure varies with distance down the magnetotail. Plasma pressures are derived from the second velocity moment of the distribution function. Details of the method may be found in Paschmann et al. [1978]. An example of the 2D moments derived from data acquired in the near-midnight magnetotail on April 12, 1978, is shown in Figure 1. Solid (dotted) curves are, from top to bottom, the ion (electron) number density, 2D temperature, flow speed, flow azimuth, and pressure. The ion pressure (bottom panel) rises between 0800 UT and 1600 UT as the ISEE 2 spacecraft moves earthward through the near-earth magnetotail plasma sheet. The time-series of data from a single inbound pass shows nonmonotonic variations of the measured pressure. For instance, a temporary decrease in pressure is observed near 1000 UT. At this time, the motion of the plasma sheet relative to the spacecraft left ISEE 2 temporarily in the tail lobe where the pressure is low. This type of temporal and dynamical fluctuation complicates the analysis of plasma sheet pressure as a function of distance down the tail. Results that are relatively unaffected by fluctuations are obtained by analyzing a large number of passes statistically.

Throughout the near-tail plasma sheet, the ion pressure dominates the electron pressure by typically an order of magnitude (see the bottom panel of Figure 1). Therefore, in calculating the bulk plasma pressure, we have neglected the contribution from elec- trons. Observations show that ions are isotropic [see Stiles et al., 1978] justifying the use of the pressure calculated from $2 D$ ion distributions, which are routinely determined by the FPE investigators.

The high temporal resolution afforded by the FPE is unnecessary for determining the large-scale average plasma sheet pressures. Consequently, we used one minute averages of the $2 \mathrm{D}$ ion pressures and of the GSM location of the spacecraft. In addition, data were tagged with the planetary geomagnetic index, $K p$, for the 3-hour interval within which measurements were taken; data were then sorted according to $K p$. We assumed that pressure variations over a limited spatial range in $Y_{G S M}$ were small and projected all observations with $-4<Y_{G S M}<4$ into the midnight meridian plane. From the averaged data, we removed all measurements obviously taken in the tail lobes (observations characterized by significantly diminished plasma pressure and number density). Our criteria for unambiguous lobe identification were number densities less than $0.05 \mathrm{~cm}^{-3}$ and/or pressures less than $10^{-11}$ dynes $/ \mathrm{cm}^{2}$. Rather than select less stringent criteria for lobe identification (e.g., number densities less than $0.1 \mathrm{~cm}^{-3}$ as in the study of Lennartsson and Shelley [1986]), we decided to account for any unremoved lobe entries statistically. As a result, we probably retain all measurements made in the plasma sheet proper; on the other hand, we undoubtedly include some observations actually taken in the lobe that do not satisfy our identification criteria.

In the quasi-static approximation $[\rho(\partial \mathrm{v} / \partial t) \rightarrow 0]$ in which we have assumed negligible convective transport of momentum $[\rho(v \cdot \nabla) \mathbf{v} \rightarrow 0]$, the ideal magnetohydrodynamic (MHD) momentum equation is

$$
\frac{\mathbf{l}}{c} \mathbf{J} \times \mathbf{B}=\nabla \cdot \mathbf{P}
$$

where $\mathbf{J}$ is the current, $\mathbf{B}$ is the magnetic field, and $\mathbf{P}$ is the plasma pressure tensor. As noted previously, magnetotail plasma pressures are isotropic [Stiles et al., 1978], therefore in quiet time magne- 

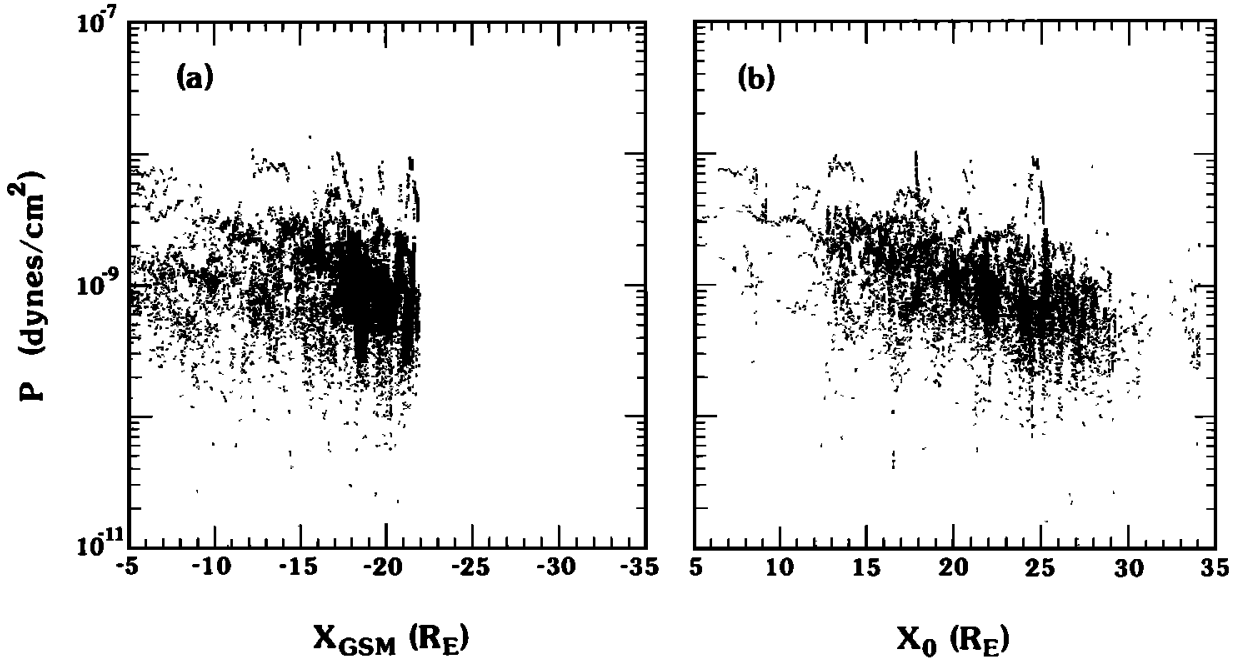

Fig. 2. Plots of plasma pressure versus location obtained by the ISEE 2 FPE during the period of March 25 to April 25, 1979. During this interval, ISEE 2 was near the midnight meridian. Each plasma pressure observation is plotted versus the $X_{G S M}$ location of ISEE 2 (panel 2a) and versus the mapped equatorial location $X_{0}$ (panel $2 b$ ). Mapping procedure is described in the text. Better ordering of the data is seen in the mapped data.

totail applications, $\nabla \cdot \mathbf{P}$ may be replaced with $\nabla P$ in equation (1). Dotting equation (1) with $B$, it is trivially shown that isotropic plasma pressure is constant along a flux tube $(B \cdot \nabla P=0)$. Assuming isotropy, we mapped off-equatorial measurements along flux tubes to the magnetic equator (i.e., the minimum B surface). We defined $X_{0}$ as the geocentric radial distance (in the midnight meridian) to the equatorial point on the field line passing through the observation location. All observations were organized by $X_{0}$. effectively the distance downtail on the equatorial surface along the midnight meridian. Note that the mapping procedure does not alter the pressure value. Rather, it maps the organizing spatial parameter from $(X, Y, Z)_{G S M}$ to $X_{0}$.

We used quantitative empirical magnetic field models developed by Tsyganenko and Usmanov [1982] (TU) to obtain $X_{0}$. These models, parameterized by the dipole tilt and the $K p$ index, are analytical fits to the average of a very large collection of in situ magnetospheric magnetic field measurements. The location, day of year, UT, and $K p$ were used as inputs to the TU models to determine $X_{0}$ for each observation.

Obviously, the accuracy of $X_{0}$ depends critically on the accuracy of the model magnetic field. Close to the Earth, the TU model represents the field quite well and $X_{0}$ is well defined. At distances greater than about $15 R_{E}$, the nomal component of the modelled equatorial field is too large when compared with observations [Tsyganenko and Usmanov, 1982]. Model field lines cross the equator too near to the Earth, yielding too small an $X_{0}$. This effect becomes more pronounced for the higher $K p$ models. The model-derived $X_{0}$ thus represents a lower limit to the actual value. Fortunately, throughout the middle magnetotail (23 $R_{E}>r>15 R_{E}$ ), the plasma sheet is relatively thin (with halfthicknesses of $\sim 3 R_{E}, \sim 2.5 R_{E}$, and 1.9-4.2 $R_{E}$ given, respectively, by Walker and Farley [1972], Bowling and Wolf [1974], and McComas et al. [1986]), so whenever ISEE 2 is in the plasma sheet, it is quite near the magnetic equator. Even an inaccurate field model introduces relatively unimportant errors when field lines need be followed for only a few degrees of latitude. Thus we believe that the TU models provide a reasonable first-order quantitative tool for describing the magnetic field in the regions studied.
As an example of our mapping procedure, in Figure 2 we show scatter plots of the measured pressures versus downtail distance. Data from ISEE 2 tail passes over a one month period between March 25 and April 25, 1979, are plotted both versus the $X_{G S M}$ location of the spacecraft $(2 a)$ and versus $X_{0}(2 b)$. A large degree of scatter is evident in both plots but the scatter is reduced in Figure $2 b$ (i.e., the range and $\mathrm{ms}$ deviation of the mapped pressures at a fixed location are smaller). For $r>15 R_{E}$, the typical ms deviation of the pressure in Figure $2 b$ is roughly $2 / 3$ of the rms deviation in Figure $2 a$. The reduction of the range in pressure is especially evident for $r<15 R_{E}$ where the data mapped using the TU models are better behaved. In this example, $14 \%(25 \%, 50 \%$, $75 \%)$ of the data were mapped by $X<0.5(1,5,9) R_{E}$, where $X=X_{0}-\left|X_{G S M}\right|$. Some of the remaining scatter arises partly because the data have not been sorted by level of magnetic activity and partly because, despite the removal of obvious lobe intervals, some entries into the tail lobes may not have been excluded. We treat these matters next.

To eliminate some of the scatter associated with activity levels, we binned the observations using an effective $K p$ index. The bin labeled $K p^{*}=i$ includes observations made in any three hour interval for which $K p=i^{-}, i$, or $i^{+}$(see analogy in Tsyganenko and Usmanov [1982]). For example, $K p^{*}=1$ corresponds to $K p=1^{-}, 1,1^{+}$. Eleven subsets $\left(K p^{*}=0 ; 0^{+} ; 1^{-} ; 1 ; 1^{+} ; 2^{-}\right.$; $2 ; 2^{+} ; 3^{-}: 3,3^{+} ;$and $>3^{+}$) were compiled, ranging from quiet to disturbed, to investigate how the pressure profiles change with magnetic activity.

Figure 3 shows pressure values plotted versus $X_{0}$ for both low $\left(K p^{*}=1\right)$ and moderate $\left(K p^{*}=2^{+}\right)$levels of magnetic activity. The range of pressure at fixed $X_{0}$ is somewhat smaller than in Figure $2 b$; however, the Ims variation of the pressure is not significantly decreased. Despite the fluctuations it is possible to observe in both cases that the plasma pressure rises by an order of magnitude between $X_{0}=35 R_{E}$ and $X_{0}=5 R_{E}$. In addition, the pressure magnitude is generally greater for the more active case, especially at smaller distances.

Some remaining scatter evident in Figure 3 is produced by unidentified transits into the tail lobes where the pressure is lower than in the bulk of the data. In addition, scatter can be produced 


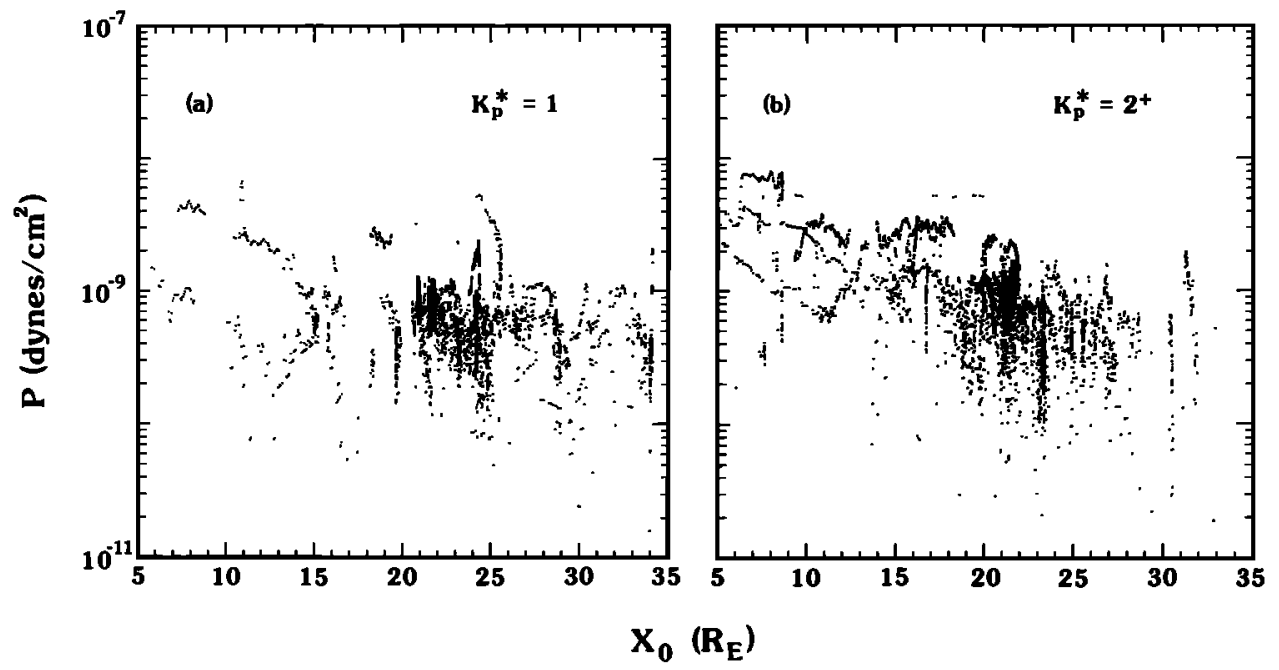

Fig. 3. Scatter plots of the plasma sheet pressure versus $X_{0}$ including data from all ISEE 2 near-midnight observation sorted according to magnetic activity; data from a low $\left(K p^{*}=1\right)$ and a moderate $\left(K p^{*}=2^{+}\right)$level subset are shown.

by variations in the solar wind dynamic pressure (see, for example, Fairfield [1987]). Similar scatter has been evident in previous statistical studies of plasma sheet pressures [Huang and Frank, 1986; Lennartsson and Shelley, 1986] where peak pressures were observed at the center of the plasma sheet where the field minimizes. In this study, we are primarily concemed with central plasma sheet particle pressure, in the region where the plasma sheet magnetic pressure is much less than the lobe magnetic pressure. To extract results for the peak central plasma sheet pressure, the data in each $K p^{*}$ subset were sorted into $0.5 R_{E}$ bins in $X_{0}$ and the median and quartile values of the pressure were determined in each bin. The $0.5 R_{E}$ bin size was chosen to assure the temporal and spatial independence of the data and is discussed below. We feel that the upper quartile pressure measurements (i.e., peak pressures) give good estimates of the actual near-equatorial plasma pressures. Figure 4 shows the median and upper and lower quartiles of the binned pressure data for the $K p^{*}=1^{-}$subset and all three traces follow the same trend, decreasing by an order of magnitude between $5 R_{E}$ and $35 R_{E}$. Henceforth, we focus on the upper quartile of the binned ion pressure as most representative of peak central plasma sheet pressure.

In order to estimate the errors in the pressure profiles, we must consider how many independent (both temporally and spatially) estimates are available in each downtail bin. Figure 5 shows the number of observations (or equivalently number of minutes spent) in each $X_{0}$ bin for the $K p^{*}=2^{+}$case, a moderate activity level. This distribution is typical. At or near $23 R_{E}$, the $X_{0}$ bins usually contain several hundred data points, while at both smaller and larger $X_{0}$ the number drops to on the order of 60 averaged observations. It should be noted that the number of independent measurements within an $X_{0}$ bin is somewhat lower than the number of cases plotted in Figure 5. For measurements to be temporally independent within any particular bin, the bin size $\left(0.5 R_{E}\right)$ should be of the same order as the scale length defined by the product of the duration of the observation $(60 \mathrm{~s})$ and the velocity along $X_{0}$. For the measurements to be spatially independent within a bin, the averaging bin size should be greater than or equal to the observation scale length. Therefore to assure both temporal and spatial independence within a bin, the bin size should be nearly the observation scale size.

When the ISEE 2 spacecraft passed through a nearly stationary plasma sheet (as in Figure 1 from 1000 to 1600 UT) the relevant speed is the orbital speed along $X_{0}$ ( $\sim 8 \mathrm{~km} / \mathrm{s}$ at these distances) which yields a size scale of $\sim 0.08 R_{E}$, smaller than the $0.5 R_{E}$ bin size. These observations, while spatially independent, are not temporally independent within a bin. Therefore one orbit could heavily bias the value within a particular bin. This generally occurred on smaller $L$ shells $\left(<12 R_{E}\right.$ ), where the number of independent measurements is roughly $1 / 6$ of the number plotted. Fortunately, more than $80 \%$ of the data are from periods when the relevant velocity is that of the plasma sheet rather than the spacecraft. These data are characterized by entries into and exits out of the plasma sheet on relatively short time scales ( $<15 \mathrm{~min}$ ) caused by plasma sheet flapping or plasma sheet recovery following a substorm. Plasma sheet motion normal to the equatorial surface

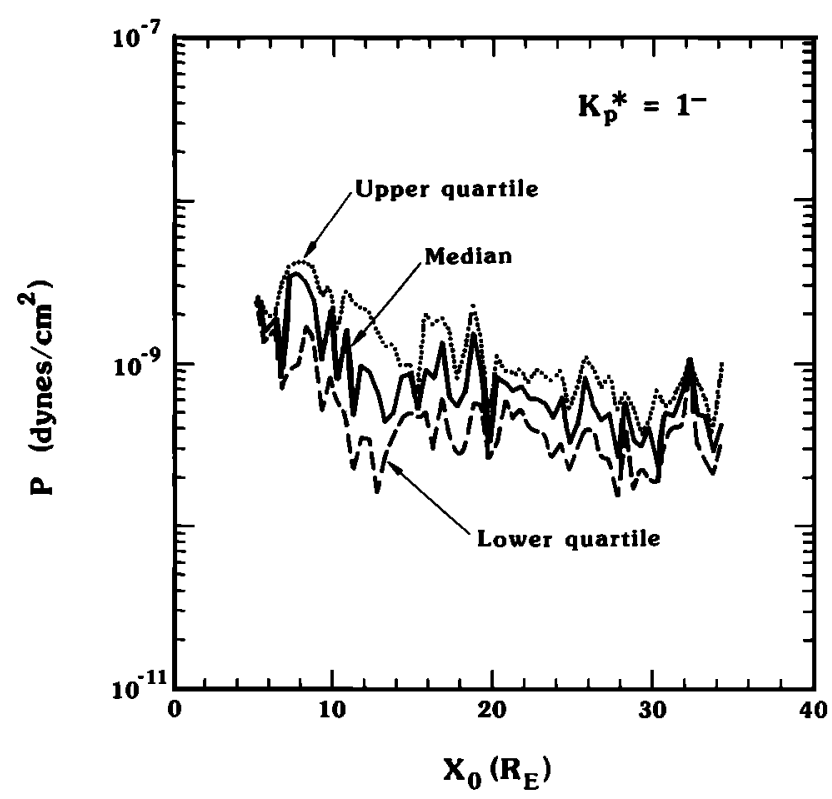

Fig. 4. The median and upper and lo /er quartiles of the binned pressure (dynes $\left.\mathrm{cm}^{-2}\right)$ versus $X_{0}\left(R_{E}\right)$ for a magnetically quiet data set $\left(K p^{*}=\right.$ $1^{-}$). The upper quartile is probably most representative of the plasma pressure near the center of the plasma sheet. 


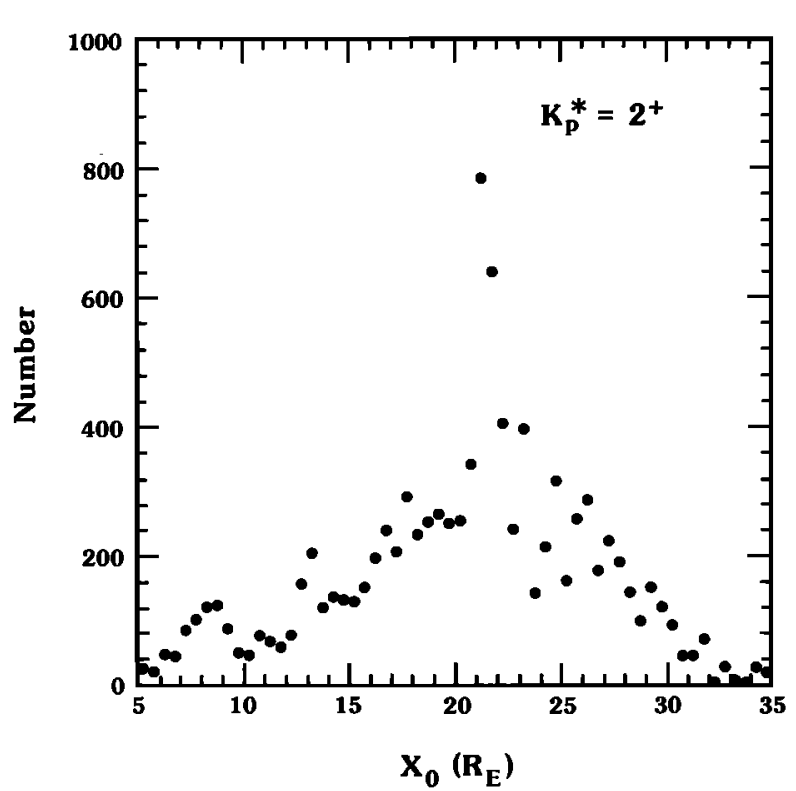

Fig. 5. The distribution of number of observation points in each $0.5 R_{E}$ $X_{0}$ bin for a moderate magnetic activity case $\left(K p^{*}=2^{+}\right)$. Typically, bins near apogee contain many hundred data points. Throughout most of the region, the number of samples constitutes a statistically significant data set for all magnetic activity levels.

displaces the spacecraft to different field lines, thus changing the effective $X_{0}$. Using the TU model, we find that, in the near-tail, a vertical displacement of $1 R_{E}$ from the center of the plasma sheet corresponds to a change of several $R_{E}$ in $X_{0}$. We choose $3 R_{E}$ as a representative value of the effective range of $X_{0}$ sampled in a plasma sheet encounter. If the $\mathbf{1 5}$ minutes duration of an encounter is divided equally between entry into and exit out of the plasma sheet, the effective velocity along $X_{0}$ is $>40 \mathrm{~km} / \mathrm{s}$. Therefore the size scale of the observations is approximately $0.4 R_{E}$ in $X_{0}$, comparable with the $0.5 R_{E}$ bin size used in Figures 4 and 5 . For this reason, we feel that outside of $\sim 12 R_{E}$ the values shown in Figure 5 approximate the number of both temporally and spatially independent measurements in each bin. Bins with at least 120 samples $\left(13<X_{0}<30\right)$ have a probable error of the mean less than $10 \%$ of the standard deviation. Beyond this range, the statistical uncertainty is much greater and the curves must be interpreted with caution.

The pressure profiles cannot be corrected for errors inherent to the mapping technique but the nature of the errors so introduced can be described and, to some extent, quantified. We define $Z$ as the distance of the spacecraft from the average position of the nentral sheet in the $Z_{G S M}$ direction. It is well known that processes within the magnetotail (one example being plasma sheet "flapping" [Hones, 1979]) cause the neutral sheet to deviate from its average location. In order to estimate the error in $X_{0}$ caused by variations in $Z$, we have nsed the TU model. At fixed $X_{G S M}$ locations, $X_{0}$ was determined for a range of $Z$ about its nominal value. Variations of $2 R_{E}$ in $Z$ (the average departure of the near-tail plasma sheet from its nominal location [Bowling and Wolf, 1974]) were assumed. Over the $\pm 2 R_{E}$ range in $Z, X_{0}$ varied typically by $<5 R_{E}$. Variations were largest for the more active models. Fortunately, the effect of mapping errors near apogee are not great because the pressure gradients versus $X_{0}$ are rather shallow (see Figure 4) at large distances.

The response of near-magnetotail pressure distributions to ch-

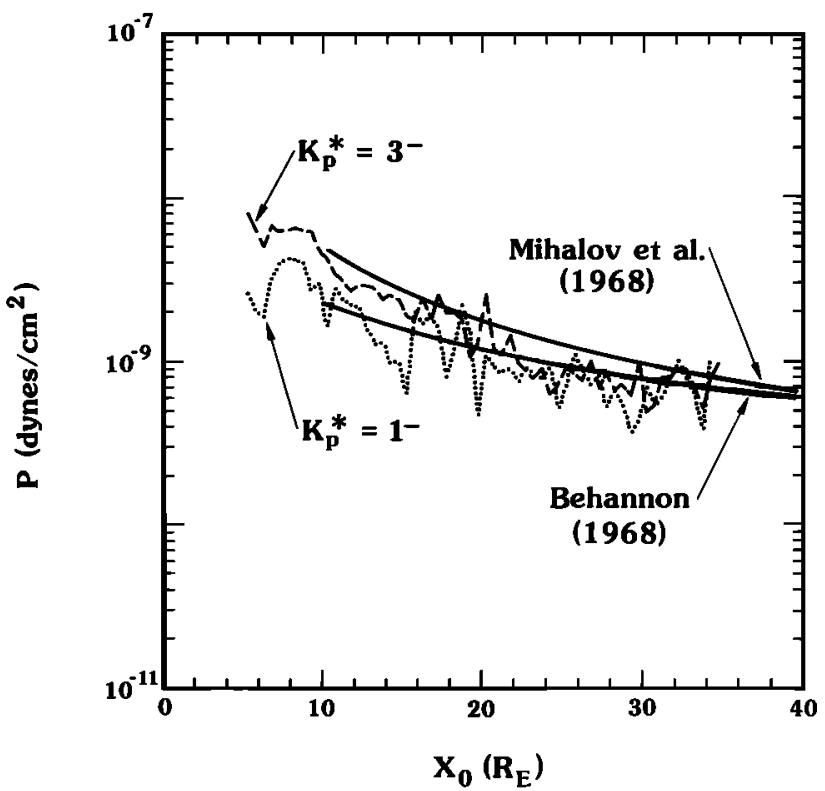

Fig. 6. The upper quartile pressure curves from both a quiet $\left(K p^{*}=1^{-}\right)$ and a disturbed $\left(K p^{*}=3^{-}\right)$data subset plotted versus $X_{0}$. The more active data set exhibits both larger pressure magnitudes and earthward gradients. Superimposed are observed lobe magnetic pressure detemined by Mihalov et al. [1968] and Behannon [1968]. The mapped plasma sheet pressures and the tail lobe magnetic pressures show similar distance dependences.

anging levels of activity is shown in Figure 6. Curves for the upper quartile of data are plotted for $\mathrm{Kp}^{*}=3^{-}$and $1^{-}$. Both the plasma sheet plasma pressure magnitudes and earthward pressure gradients are larger in the data for more active periods. Between the outer boundary of the analysis and $10 R_{E}$, the plasma pressure rises by an order of magnitude for both activity levels with the bulk of the increase occurring inside of about $20 R_{E}$. The actual rise in pressure is undoubtedly even greater than shown because the fraction of the energy density contributed by particles whose energy falls above the FPE range increases with decreasing distance. Thus the plotted pressure approximates the actual plasma pressure only in regions where the bulk of the energy density is measured by the FPE detector. We have examined representative FPE ion dynamic differential energy spectra and find that for $X_{0}$ near $15 R_{E}$, ions with energies between a few and $\sim 20 \mathrm{keV}$ provide the bulk of the energy density. As the spacecraft moves earthward, the peak of the energy density shifts to higher energies as would occur for adiabatic convection. Eventually, the peak of the energy density approaches the uppermost energy channel of the FPE and a substantial fraction of the distribution goes undetected. Thus inside of $\sim 12 R_{E}$, the pressures in Figure 6 are lower limits, and inward gradients are much steeper.

Beyond about $25 R_{E}$, the two distributions are approximately the same in magnitude and appear to be fairly constant with distance. This similarity must be taken with some skepticism because of problems with the model field beyond $25 R_{E}$ (discussed earlier) as well as because of the scarcity of data at these distances. In addition, data presented by Baumjohann et al. [1988] suggest that the characteristic plasma energy at a given $X_{0}$ increases during active times (see also Lennartsson and Shelley [1986] and Huang and Frank [1986]). Therefore it is possible that the flux at energies above the highest energy channel of the FPE is significant much farther down the tail during intervals of high $K p$. Evidently, this 
TABLE 1. Explorer 45 Proton Preseure (dynes $\mathrm{cm}^{-2}$ ) for $2100<L T<2400$

\begin{tabular}{ccccc}
\hline $\begin{array}{c}L \\
R_{E}\end{array}$ & $\begin{array}{c}\text { Quiet } \\
(1-872 \mathrm{keV})\end{array}$ & $\begin{array}{c}\text { Moderate } \\
(1-872 \mathrm{keV})\end{array}$ & $\begin{array}{c}\text { Disturbed } \\
(1-872 \mathrm{keV})\end{array}$ & $\begin{array}{c}\text { Moderate } \\
(1-24 \mathrm{keV})\end{array}$ \\
\hline 2.5 & $2.7 \times 10^{-8}$ & $3.3 \times 10^{-8}$ & $4.0 \times 10^{-8}$ & $4.0 \times 10^{-9}$ \\
3.0 & $7.3 \times 10^{-8}$ & $8.0 \times 10^{-8}$ & $1.7 \times 10^{-7}$ & $2.3 \times 10^{-9}$ \\
3.5 & $8.0 \times 10^{-8}$ & $1.1 \times 10^{-7}$ & $2.3 \times 10^{-7}$ & $2.3 \times 10^{-9}$ \\
4.0 & $9.3 \times 10^{-8}$ & $1.0 \times 10^{-7}$ & $1.9 \times 10^{-7}$ & $6.0 \times 10^{-9}$ \\
4.5 & $6.7 \times 10^{-8}$ & $9.3 \times 10^{-8}$ & $1.5 \times 10^{-7}$ & $1.4 \times 10^{-8}$ \\
5.0 & $4.3 \times 10^{-8}$ & $5.7 \times 10^{-8}$ & $1.0 \times 10^{-7}$ & $6.0 \times 10^{-9}$ \\
5.5 & $3.3 \times 10^{-8}$ & $5.0 \times 10^{-8}$ & $8.0 \times 10^{-8}$ & $5.3 \times 10^{-9}$ \\
\hline
\end{tabular}

After Smith and Hoffman [1973].

would cause us to underestimate the plasma pressure, but we are unable to quantitatively assess the effect on the $K p=3^{-}$curve.

The curves of Figure 6 contain direct information of the timeaveraged plasma pressure in the plasma sheet, and indirect information of the magnetic pressure in the adjacent tail lobes. In the limit of ideal MHD, where we assume an isotropic plasma, equation (1) can be written in the form

$$
\nabla\left(P+\frac{B^{2}}{8 \pi}\right)=\frac{1}{4 \pi}(B \cdot \nabla) B
$$

where gradients in the total plasma and magnetic pressure are balanced by magnetic tension $\left(\frac{1}{4 \pi}(B \cdot \nabla) B\right)$. The two-dimensional tail approximation is characterized by the ordering scheme (for a more complete discussion see Schindler and Birn [1986])

$$
B_{x}, \frac{\partial}{\partial z}=O(1) \quad B_{y}, B_{z}, \frac{\partial}{\partial x}, \frac{\partial}{\partial y}=O(\epsilon)
$$

When the tail approximation (equation (3)) applies, it can be show that through $O(\epsilon)$ the $z$ component of equation (2) reduces to

$$
\nabla\left(P+\frac{B^{2}}{8 \pi}\right)=0
$$

In the tail lobes, the magnetic pressure is much greater than the plasma pressure and at the center of the plasma sheet, the plasma pressure dominates the magnetic pressure (see, Fairfield $e t$ al. [1981] and Fairfield [1987]). Therefore integration of equation (4) in the $z$ direction yields the approximate requirement that

$$
P_{p s}=B_{l}^{2} /(8 \pi)
$$

where $P_{p s}$ is the central plasma sheet plasma pressure and $B_{l}$ is the asymptotic lobe magnetic field strength. Regions in which equation (5) applies, are those where $\nabla\left(B^{2} / 8 \pi\right)>>\frac{1}{4 \pi}(\mathbf{B} \cdot \nabla) B$ and we use the TU models to test the inequality. We determined that equation (5) is valid for $X_{0}$ greater than $\sim 15 R_{E}$, where gradients of the magnetic pressure are at least ten times greater than the magnetic tension.

The average lobe magnetic field as a function of $X_{0}$ is known from the work of Mihalov et al. [1968] and Behannon [1968]. Their fits to hourly averaged Explorer 33 data were used to obtain two curves of the lobe magnetic pressure superimposed on Figure 6; curves are plotted only for $X_{0}>15 R_{E}$ where equation (5) applies. The study by Mihalov $e t a l$. included data from slightly higher magnetic activities $\left(K p<2^{+}\right)$than did the Behannon work $(K p<2)$. The curves representing the lobe magnetic and plasma sheet plasma pressures follow each other reasonably closely in to $15 R_{E}$. Inside of $\sim 15 R_{E}$, the balance of magnetic and thermal pressures must be described by equation (2) rather than equation (5). In fact, if the pressure is anisotropic, the relevant balance is expressed by equation (1). Solutions of the latter type, obtained by Spence et al. [1986], are discussed in a following section.

In our discussion of Figure 6 we noted that the plasma pressures are underestimated by FPE measurements inside of $\sim 12 R_{E}$. Consequently, we tum to other data to supplement FPE measurements inside of $12 R_{E}$. The three sources of supplementary data, listed in order of increasing apogee distance, are the Explorer 45 $\left(S^{3} A\right)$ satellite, the ATS 5 satellite, and the AMPTE CCE satellite.

\subsection{Explorer $45\left(S^{3} A\right)$ Plasma Experiment}

The Small Scientific Satellite $\left(S^{3} A\right)$, Explorer 45 , was launched into an elliptical, equatorial orbit with its apogee at $5.24 R_{E}$. Particle measurements were made with channel multiplier detector systems and solid state detectors which measured ions with energies between $800 \mathrm{eV}$ and $>3.8 \mathrm{MeV}$. Smith and Hoffiman [1973] used these data to characterize the proton pressure in the equatorial magnetosphere for $L$ shells interior to $5.5 R_{E}$. Smith and Hoffman analyzed ion measurements in the $1-872 \mathrm{keV}$ range obtained on December 16-18, 1971, when apogee was near 2100 hours magnetic local time. They presented data from several orbits spanning a wide range of magnetic activities. From their published results, the ion pressure as a function of radial distance and level of magnetic disturbance along the midnight meridian may be roughly estimated. We restrict our attention to intervals when the spacecraft was near apogee and was within $\sim 3$ hours of midnight. We justify the assumed independence of local time by appeal to the work of DeForest and McIlwain [1971] who showed that for ions, with energies between $50 \mathrm{eV}$ and $50 \mathrm{keV}$ at geostationary orbih the typical observed pressure varied by only about $10 \%$ between $2100 \mathrm{LT}$ and $0000 \mathrm{LT}$.

Table 1 tabulates pressures from Smith and Hoffiman [1973] for three orbits representing quiet (prolonged geomagnetically quiescent conditions), moderate (growth phase of an isolated substorm), and disturbed (recovery phase of a large storm) conditions. Data were taken in $0.5 R_{E}$ radial bins, for each of the three activity levels. The second through fourth columns contain pressures determined over energies of 1 to $872 \mathrm{keV}$. Pressures determined with the lowest energy channel ( 1 to $24 \mathrm{keV}$ ) during moderate conditions are listed in the last column. Between 5.5 and $3.5 R_{E}$, the pressure increases by a factor of 2 to 3 , with a peak between 3.5 and $4.5 R_{E}$. Interior to about $3.5 R_{E}$ the pressure declines rapidly. At $2.5 R_{E}$, the pressure has fallen below its value at $5.5 R_{E}$. The observed pressure is a strong function of substorm 
TABLE 2 ATS 5 Proton Pressure $(50 \mathrm{eV}$ to $50 \mathrm{keV})$ at Geosynchronous Orbit

\begin{tabular}{cccc}
\hline $\begin{array}{c}\text { Local } \\
\text { Time, } \\
\text { hours }\end{array}$ & $\begin{array}{c}\text { Minimum } \\
\text { Pressure, } \\
\text { dynes cm-2 }\end{array}$ & $\begin{array}{c}\text { Typical } \\
\text { Pressure, } \\
\text { dynes cm }\end{array}$ & $\begin{array}{c}\text { Maximum } \\
\text { Pressure, } \\
\text { dynes cm }\end{array}$ \\
\hline 2100 & $5.6 \times 10^{-9}$ & $1.2 \times 10^{-8}$ & $3.3 \times 10^{-8}$ \\
0000 & $6.6 \times 10^{-9}$ & $1.4 \times 10^{-8}$ & $2.4 \times 10^{-8}$ \\
0300 & $5.1 \times 10^{-9}$ & $1.2 \times 10^{-8}$ & $2.0 \times 10^{-8}$ \\
\hline
\end{tabular}

From DeForest and Mcllmain [1971].

activity. In each radial bin of Table 1 , the pressure increases by about a factor of 2 between the quietest and the most active period.

Table 1 confirms that at $\sim 5 R_{E}$ the FPE measurements underestimate the total pressure by at least an order of magnitude for both the quiet and the more active data sets. Within the range of energies measured by both FPE and the Explorer 45 detector, the partial pressures are consistent with each other. In particular, pressures derived from the Explorer 45 1-24 keV energy channel (see Table 1, column 5) may be compared with pressures obtained from the FPE (70 eV to $40 \mathrm{keV}$ ). During moderate activity at $5 R_{E}$, the FPE yielded pressures of $\sim 8 \times 10^{-9}$ dynes $/ \mathrm{cm}^{2}$ whereas the lowest energy Explorer 45 partial pressures were $6 \times 10^{-9}$ dynes $/ \mathrm{cm}^{2}$; the difference can be attributed to the FPEs slightly broader energy coverage. The Smith and Hoffman data show that at $\sim 5 R_{E}$ the fraction of the pressure carried by the 1 to $24 \mathrm{keV}$ ions is relatively small ( $<10 \%$ to $\sim 30 \%$ of the total). Therefore while the FPE pressures are probably quite reasonable beyond about $12 R_{E}$, they are too low by $\sim 70 \%$ to $>90 \%$ in the inner regions $\left(\sim 5 R_{E}\right)$. The data require that very steep pressure gradients must exist in the "transition" zone linking the relatively low pressure region beyond $12 R_{E}$ with the high pressure region at distances less than $\sim 5 R_{E}$.

\subsection{ATS 5 UCSD Plasma Experiment}

Further insight into the near-tail pressure gradient can be gained using plasma data obtained from geostationary spacecraft. The average plasma pressure at $\sim 6.6 R_{E}$ was characterized by DeForest and McIlwain [1971] using data from the ATS 5 University of California at San Diego (UCSD) plasma experiment. Protons and electrons with energies ranging from $50 \mathrm{eV}$ to $50 \mathrm{keV}$ were detected.

DeForest and McIlwain found that although the structure of the energy spectra was rather variable, the integrals of the spectra at any given magnetic local time were much more stable. For 29 days in January 1970, they determined the minimum, maximum, and typical values of the plasma pressure in six different local time bins. The results for ion pressure in the three near-midnight local time bins from Table 1 of their paper are reproduced here in Table 2. The bins are centered at the noted times. Pressures tabulated in Table 2 vary by roughly 75\% with activity. Peak pressures occur in the midnight local time bin except for the most active times when the peak moves duskward. For quiet and typical periods, the average pressures vary very little $(<20 \%)$ within six hours centered about midnight.

Comparison of the ATS 5 pressures with the Explorer 45 data suggests that the pressure decreases by a factor of 7 to 8 between 5.5 and 6.6 $R_{E}$. However, as DeForest and Mcllwain [1971] point out, pressures determined from the ATS 5 plasma data are lower limits as the moments were taken only over the range of $\mathbf{5 0}$ $\mathrm{eV}$ to $50 \mathrm{keV}$. This means that the combined data of Tables 1 and
2 may overestimate the steepness of the inward pressure gradient inside of geostationary orbit.

\subsection{AMPTE CCE Plasma Experiments}

In recent years, great progress has been made in determining both the energy distribution and composition of particles in the inner magnetosphere with measurements from particle detectors on the charge composition explorer (CCE) of the active magnetospheric particle tracer explorers (AMPTE) mission. CCE's apogee is 8.8 $R_{E}$ and therefore provides data in the regions surrounding geostationary orbit where systematic studies have been wanting. Unfortunately, there have been no published results of pressures near midnight. However, with appropriate assumptions and some measure of caution, observations at other local times can be used to infer plasma pressures near midnight. Below, we summarize the available observations away from midnight and then describe how they were mapped to the midnight meridian.

Studies of the evolution of the ring current plasma during the September 4-7, 1984, magnetic storms have used the medium energy particle analyzer (MEPA) [see McEntire et al., 1985, and Lui et al., 1987]. Data were obtained by an ion head on the MEPA which provided total energy measurements for ions with energies between $\sim 25 \mathrm{keV}$ and $1 \mathrm{MeV}$. Pressure moments in both studies were calculated assuming that all detected ions were protons. Data were obtained on an inbound pass between $\sim 8.5 R_{E}$ (1500 LT) and $\sim 2.5 R_{E}$ (1700 LT). In the Lui et al. [1987] study, both magnetically quiet and disturbed pressure profiles were determined. The quiet profiles were obtained during periods of reasonably small Dst $(<|10| \mathrm{nT})$. Representative examples of the disturbed (solid curve) and quiet (dashed curve) pressure profiles are reproduced from Lui et al. [1987] in Figure 7. As before, the pressure reaches a maximum of almost $2 \times 10^{-7}$ dynes $/ \mathrm{cm}^{-2}$ at 3-4 $R_{E}$. Generally, during more disturbed periods the pressure is roughly double that of quiet periods.

To make use of the Lui et al. results for local times between 1500 and 1700 LT for this investigation of pressure profiles near midnight, we mapped the measured fluxes to local midnight along constant $B$ contours. To find out if the constant $B$ mapping is a good assumption, we considered how drift paths would change in the presence of a nominal cross-tail electric field. Lui et al. [1987] show that for the cases they studied, a pancake type pitch angle distribution (i.e., peaked at $90^{\circ}$ pitch angles) of energetic ions was dominant in the ring current region. Therefore we calculated the drift paths of $90^{\circ}$ pitch angle protons between $25 \mathrm{keV}$ and 1 $\mathrm{MeV}$ assuming time independent dipole magnetic field, a $65 \mathrm{kV}$ cross-tail electric potential and a corotation electric field. In the inner magnetosphere ( $L=2.5$ to 6.5 ), the $90^{\circ}$ protons drift from 1600 LT to midnight on orbits that deviate from paths at constant $L$ by less than $8 \% / 3 \% / 1 \%$ at $25 / 75 / 150 \mathrm{keV}$. Exterior to $\sim 6.5$ $R_{E}$, the mapping at constant $B$ is no longer valid because electric 


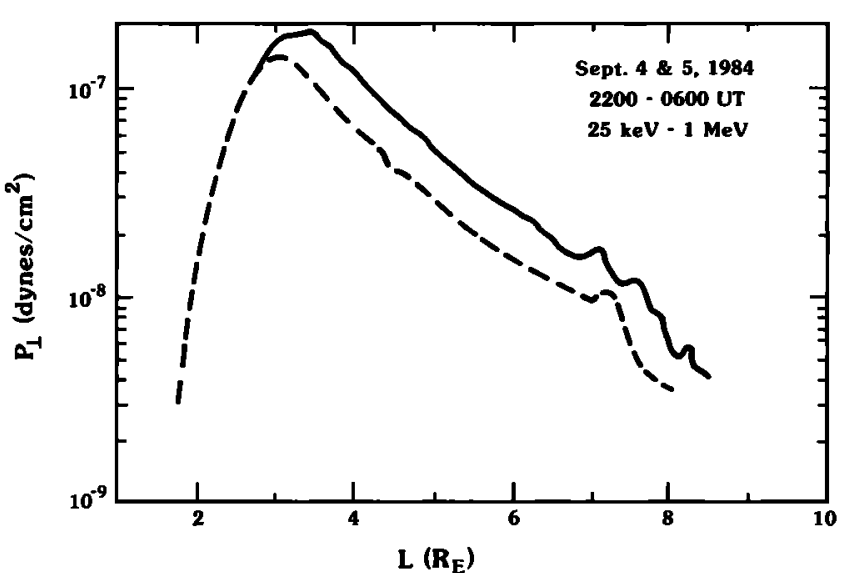

Fig. 7. The radial profiles of the particle pressure perpendicular to the magnetic field during the September 4-7, 1984 stom, taken between 1500 LT $\left(8.5 R_{E}\right)$ and $1700 \mathrm{LT}\left(\sim 3 R_{E}\right)$. The dashed curve is from a quiet reference pass obtained before the commencement of the magnetic stom (adapied from Lui et al. [1987]).

drifts become comparable with magnetic drifts. For instance, for a dipole field, the deviation from constancy approaches $20 \%$ for the $25 \mathrm{keV}$ protons near the spacecraft apogee of $8.5 R_{E}$. Realistic drift orbits carry particles toward larger $B$ at midnight and reduce the pressure. This means that interior to synchronous orbit the mapping is probably quile good while at greater distances the mapping yields a lower limit on the pressure.

\section{Pressure Profiles in the Midnight Meridian BETWEEN 2.5 AND $35 R_{E}$}

In Figure 8 we provide plots of the plasma measurements discussed above. The Lui et al. [1987] AMPTE CCE observations have been mapped to the midnight meridian by using the TU models and the assumption $B=$ constant. Both the quiet and active pressure profiles are included. As the TU field is realistically compressed on the dayside and stretched on the nightside, constant $|B|$ contours move earthward between local afternoon and midnight. The effect is to shift near-apogee (8.5 $R_{E}$ ) AMPTE CCE measurements at $\sim 1600 \mathrm{LT}$, to $\sim 7 R_{E}$ on the midnight meridian. The tabulated pressures derived from Explorer 45 (in Table 1) and ATS 5 (in Table 2), as well as the ISEE 2 FPE pressures (from Figure 6), are also shown. For distances less than $12 R_{E}$, the FPE provides only lower limits to the plasma pressure, so the curves of Figure 6 have been terminated at $12 R_{E}$.

Figure 8 provides our best estimate of the peak plasma pressure profile in the midnight meridian of the magnetotail. For $X_{0}>12 R_{E}$, the ISEE 2 FPE samples enough of the ion distribution function to yield representative plasma pressures for quiet and perhaps, more disturbed conditions. In the inner magnetosphere ( $2.5<X_{0}<5.5 R_{E}$ ), the Explorer 45 measurements are quite reliable. The plotted points represent the typical (moderately active) observed pressures; bars range from the maximum (disturbed) to the minimum (quiet) reported values. The AMPTE CCE measurements between 2.5 and $4 R_{E}$ are consistent with the Explorer 45 measurements for both quiet and disturbed conditions. As the two instruments sample different ranges of energy, the agreement suggests that the characteristic energy in this spatial range is above $25 \mathrm{keV}$. Williams [1980] found that for $3.6<L<4.2,50 \%$ of the integral ring current pressure resided in the $>85 \mathrm{keV}$ protons, consistent with the above arguments. Between 4 and $5.5 R_{E}$, the AMPTE CCE pressures fall below the Explorer 45 values by roughly a factor of 2 . We infer that much of the plasma pressure in this region is carried by particles whose energy is near the 25 $\mathrm{keV}$ threshold of the CCE detector. Measurements made at synchronous orbit by the ATS 5 UCSD experiment are comparable to the AMPTE CCE observations at the same distance. Because the ATS 5 experiment sampled much lower energies than AMPTE CCE, particles with energies less than $\sim 25 \mathrm{keV}$ must not contribute significantly to the pressure at synchronous orbit. Outside of synchronous orbit, CCE probably provides a lower bound to the total pressure as the peak of the pressure moves to lower energies. Note that the CCE curves taken from Figure 7 are terminated at 6.6 $R_{E}$ in Figure 8. In sum, the combined ATS 5, AMPTE CCE, and Explorer 45 data yield a reasonably accurate plasma pressure profile, from geostationary orbit to $2.5 R_{E}$, in the midnight meridian.

\section{Discussion AND SUMmaRY}

The profile of particle pressure along the midnight meridian at quiet and disturbed times has been obtained by using measurements from many spacecraft. The pressure changes are relatively gentle except in a region between 5 and $12 R_{E}$ where data coverage is incomplete. We refer to this region as the "transition" region.

Previous discussion of the distribution of currents and plasma in the transition region should be noted. Spence et al. [1987] inferred the distribution of plasma in the near-magnetotail by assuming magnetohydrostatic equilibrium within the TU magnetospheric magnetic field models. The region in which the model field was applicable was nearly coincident with the "transition" region $(6.5<L<12)$. In Figure 8, we have superimposed the Spence et al. $K p^{*}=0$ and $K p^{*}>3^{+}$solutions. The model-derived

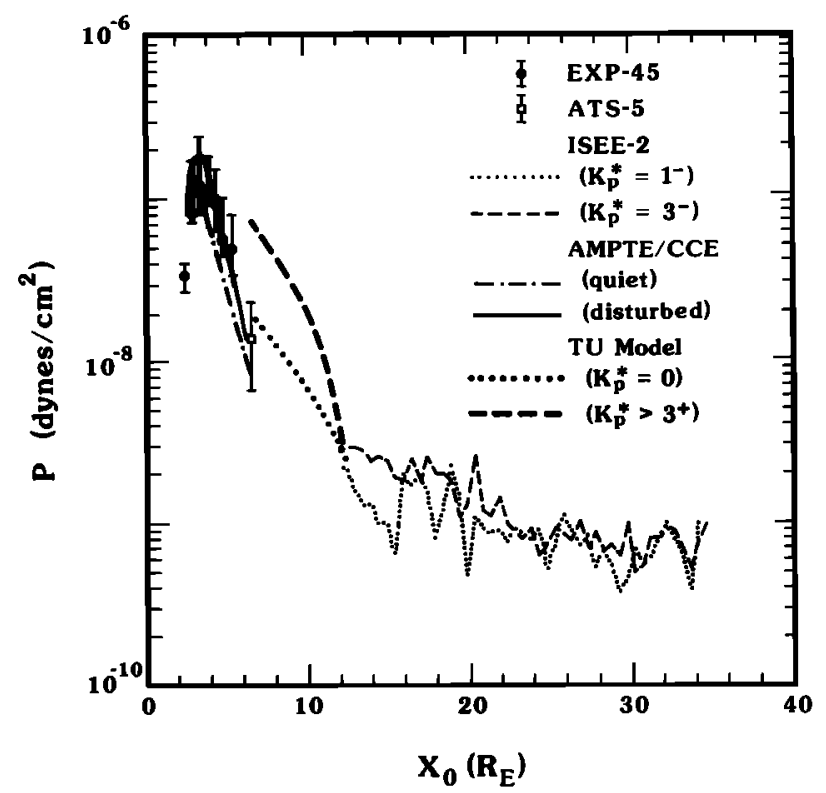

Fig. 8. A composite of the ISEE data from Figure 6, the Explorer 45 data in Table 1, the ATS 5 data in Table 2, the Lui et al. [1987] data of AMPTE CCE mapped from dusk (as in Figure 7) to midnight, and the $K p^{*}=0$ and $K p^{*}>3^{+}$pressure curves determined by "inverting" the TU magnetic field models (taken from Spence et al. [1987]). The "transition" region, where the magnetic field topology changes from dipolar to tail-like, coincides with the region $\left(7-12 R_{E}\right)$ where spacecraft measurements of plasma pressure are not available. 
pressures bridge the gap between observations on either side of the transition zone in a reasonable way; both the magnitude of the inferred pressure and the pressure gradient agree quantitatively with the data. The agreement is especially good inside of $10 R_{E}$ where the solutions have smaller errors. Typically, the model solutions satisfy the magnetostatic condition to within $\sim 10 \%$. These small errors are probably responsible for the unconvincing second derivative of the model-derived pressure profiles (particularly beyond $10 R_{E}$ ). We emphasize that the solutions obtained from the higher $K p$ models have larger associated errors than do the less active models. Not only is the model field less representative of an actual instantaneous field configuration, but also during more active intervals the magnetotail is less likely to be well-described by equation (1). In a future paper, we will more thoroughly compare the inferred pressures from the less active models with the data presented herein.

The plasma pressures obtained in the present paper may serve as a guide for equilibrium tail models such as those developed by Birn [1987]. The Birn model requires both equatorial field and plasma pressure profiles in the near tail which serve as boundary conditions to the full 3D equilibrium solution. Knowledge of plasma pressure data may also be useful in studies of wave properties in the inner magnetosphere. Moore et al. [1987] used empirical models of field and plasma characteristics to deduce the variations of the Alfven and fast mode wave speed in the magnetosphere. By combining empirical number densities with the pressures deduced in the present paper, the "effective" temperature required by Moore et al. could be better constrained.

Our study of plasma pressure reveals that a significant portion of the transition region between geostationary orbit and $12 R_{E}$ near midnight is not well constrained by direct observation. At the inner edge of the transition region (synchronous orbit), comparison of pressures obtained by spacecraft with different energy ranges indicates that ions with energies less than $\sim 25 \mathrm{keV}$ do not contribute significantly to the pressure. It is clear that the pressure must fall by about an order of magnitude in the transition region in order to be consistent with both Explorer 45 at $5.5 R_{E}$ and ISEE 2 observations at $12 R_{E}$. Within the transition region, the magnetic field also undergoes striking changes, having a relatively dipolar structure at the inner edge and a distended tail-like structure at the outer edge. As well, throughout the transition region earthward convecting plasma is diverted in the azimuthal direction. Injection fronts and other important signatures of substorm-associated processes are observed in this zone. The transition region appears to be worthy of more thorough investigation than it has had to this time.

Acknowledgments. We wish to thank G. Paschmann and J. Gosling for several helpful discussions. We also wish to thank David P. Stem for supplying us with the coded version of the TU magnetic field models. This work was partially supported by the National Science Foundation, division of Atmospheric Sciences, under grants ATM 83-00523 and ATM 86-10858. One of the authors (H.E.S) was supported by an Office of Naval Research graduare fellowship. Work done at Los Alamos National Laboratory was performed under the auspices of the United States Department of Energy with support from NASA under S-04039.

The Editor thanks S. P. Christon and R. P. Lepping for their assistance in evaluating this paper.

\section{REFERENCES}

Bame, S. J., J. R. Asbridge, H. E. Felthauser, J. P. Glore, G. Paschmann, $P$. Hiemmerich, $K$. Lehmann, and H. Rosenbauer, ISEE 1 and ISEE 2 fast plasma experiment and the ISEE 1 solar wind experiment, IEEE Trans. Geosci. Elec., GE-16, 216, 1978.
Baumjoham, W., G. Paschmann, N. Sckopke, C. A. Cattell, and C. W. Carlson, Average ion moments in the plasma sheet boundary layer, $J$. Geophys. Res., 93, 11,507, 1988.

Behannon, K., Mapping the Earth's bow shock and magnetic tail by Explorer 33, J. Geophys. Res., 73, 907, 1968.

Bim, J., Magnetotail equilibrium theory: The general three-dimensional solution, J. Geophys. Res., 92, 11,101, 1987.

Bowling, S. B., and R. A. Wolf, The motion and magnetic structure of the plasma sheet near $30 R_{E}$. Planet. Space Sci. 22, 673, 1974.

DeForest, S. E., and C. E. Mcllwain, Plasma clouds in the magnetosphere, J. Geophys. Res., 76, 3587, 1971.

Fairfield, D. A., Structure of the geomagnetic tail, in Magnetotail Physics, edited by A. T. Y. Lui, JHU Press, Baltimore, Md, 1987.

Fairfield, D. H., R. P. Lepping, E. W. Hones, Jr., S. J. Bame, and J. R. Asbridge, Simultaneous measurements of magnetotail dynamics by IMP spacecraft, J. Geophys. Res., 86, 1396, 1981.

Hones, E. W., Jr., Transient phenomena in the magnetotail and their relation to substorms, Space Sci. Rev., 23, 393, 1979.

Huang, C. Y., and L. A. Frank, A statistical study of the central plasma sheet: Implications for substom models, Geophys. Res. Lett., 13, 652, 1986.

Lennarsson, W., and E. G. Shelley, Survey of 0.1 to $16-\mathrm{keV} / \mathrm{e}$ plasma sheet ion composition, J. Geophys. Res., 91, 3061, 1986.

Lui, A. T. Y., R. W. McEntire, and S. M. Krimigis, Evolution of the ring current during two geomagnetic stoms, J. Geophys. Res., 92, 7459, 1987.

McComas, D. J., C. T. Russell, R. C. Elphic, and S. J. Bame, The nearearth cross-tail current sheet: Detailed ISEE 1 and 2 case studies, $J$. Geophys. Res., 91, 4287, 1986.

McEntire, R. W., A. T. Y. Lui, S. M. Krimigis, and E. P. Keath, AMPTE CCE energetic particle composition measurements during the September 4, 1984, magnetic storm, Geophys. Res. Lett., 12, 317, 1985.

Mihalov, J. D., D. S. Colbum, R. G. Currie, and C. P. Sonetr, Configuration and reconnection of the geomagnetic tail, J. Geophys. Res., 73, 943, 1968.

More, T. E., D. L. Gallagher, J. L. Horwitz, and R. H. Comfort, MHD wave breaking in the outer plasmasphere, Geophys. Res. Lett., 14, 1007, 1987.

Paschmann, G., N. Sckopke, J. Papamastorakis, S. J. Bame, J. R. Asbridge, J. T. Gosling, E. W. Hones, Jr., and E. R. Tech, ISEE plasma observations near the subsolar magnetopause, Space Sci. Rev., 22, 717. 1978.

Schindler, K., and J. Bim, Magnetotail theory, Space Sci. Rev., 44, 1986. Smith, P. H., and R. A. Hoffman, Ring current particle distributions during the magnetic storms of December 16-18, 1971, J. Geophys. Res., 78, 4731, 1973.

Spence, H. E., M. G. Kivelson, and R. J. Walker, Static magnetic field models consistent with nearly isotropic plasma pressure, Geophys. Res. Lett., 14, 872, 1987.

Stiles, G. S., E. W. Hones, S. J. Bame, and J. R. Asbridge, Plasma sheet pressure anisotropies, J. Geophys. Res., 83, 3166, 1978.

Tsyganenko, N. A., and A. V. Usmanov, Determination of the magnetospheric current system parameters and development of experimental geomagnetic field models based on data from IMP and HEOS satellites, Planet. Space Sci. 30, 985, 1982.

Walker, R. J., and T. S. Farley, Spatial distribution of energetic plasma sheet electrons, J. Geophys. Res., 77, 4650, 1972.

Walker, R. J., and D. J. Southwood, Momentum balance and flux conservation in model magnetospheric magnetic fields, J. Geophys. Res., 87, $7460,1982$.

Williams, D. J., Ring current composition and sources, in Dynamics of the Magnetasphere, edited by S.-I. Akasofu, p. 407, D. Reidel, Hingham, Mass., 1980.

M. G. Kivelson, H. E. Spence, and R. J. Walker, Institute of Geophysics and Planetary Physics, Slichter Hall, University of California, Los Angeles, CA 90024-1567.

D. J. McComas, Los Alamos National Laboratory, Los Alamos, NM 87545.

(Received July 12, 1988;

revised November 28, 1988; accepted November 28, 1988.) 